\title{
Use of SGLT2 inhibitors during Ramadan: a survey of physicians' views and practical guidance
}

\author{
SALEM A BESHYAH, ${ }^{1}$ SUDESNA CHATTERJEE, ${ }^{2,3}$ MELANIE J DAVIES2,3
}

\begin{abstract}
Background and Objectives: SGLT2 inhibitors are a new glucose-lowering therapy for type $\mathbf{2}$ diabetes. There are no recommendations for their use for patients fasting for Ramadan.

Methods: We surveyed physicians regarding management of people with type 2 diabetes on SGLT2 inhibitors intending to fast for Ramadan.

Results: Most of the responding 197 physicians had substantial experience with patients who fast for Ramadan, with $18.6 \%$ having $25-50 \%$ such patients, $18.2 \%$ having $50-75 \%$ and $23.6 \%$ having $>75 \%$. Most physicians were fully confident $(49.4 \%)$ or fairly confident (34.4\%) about diabetes management during Ramadan. Risks of hypoglycaemia (98.1\%) and dehydration $(\mathbf{8 3 . 1} \%)$ were identified more often than hyperglycaemias $(67.5 \%)$, diabetic ketoacidosis $(63.8 \%)$ and thromboembolic disease $(45.2 \%)$. The majority felt that SGLT2 inhibitors were generally appropriate and safe during Ramadan but should be discontinued in selected patients (70.6\%). Most respondents (92.2\%) would advise taking an SGLT2 inhibitor with the first evening meal (Iftar), but 6.1\% advised taking them before the last pre-dawn meal (Suhour). Taking extra clear fluids in the evening of Ramadan was recommended by the majority.

Conclusions: This survey will help to inform interpretation of future studies towards the development of clinical guidelines for SGLT2 inhibitor use during Ramadan. The majority of physicians believe that SGLT2 inhibitors are generally safe for patients with diabetes who intend to observe Ramadan fasting, but should be discontinued for selected patients. We provide practical advice for physicians in this setting.

Br J Diabetes 2016;16:20-24
\end{abstract}

Sheikh Khalifa Medical City, Abu Dhabi, United Arab Emirates University of Leicester, Diabetes Research Centre, Leicester, UK

University Hospitals of Leicester NHS Trust, Leicester, UK

Address for correspondence: Dr Salem A Beshyah Consultant Physician, Sheikh Khalifa Medical City, Abu Dhabi, United Arab Emirates.

Tel: 00971505662723

E-mail: Beshyah@yahoo.com

http://dx.doi.org/10.15277/bjd.2016.058
Key words: SGLT2 inhibitors, Ramadan, dehydration, type 2 diabetes.

\section{Introduction}

SGLT2 inhibitors are a new class of glucose lowering therapy for type 2 diabetes that improves glycaemic control, with additional benefits relating to reduced weight and blood pressure. ${ }^{1}$ Theoretical concerns have been expressed regarding potential side-effects of SGLT2 inhibitors in patients observing the Ramadan fast, particularly in relationship to dehydration, hyponatraemia and postural hypotension. Elderly patients, those fasting for long hours and those on multiple medications for hypertension may be of particular concern. The US Food and Drug Administration (FDA) has recently issued a warning regarding diabetic ketoacidosis (DKA) associated with SGLT2 inhibitor use and a review of the issue by the European Medicines Agency is in progress. ${ }^{2,3}$ It is not known whether the use of an SGLT2 inhibitor during Ramadan increases the risk of DKA.

Although general recommendations for diabetes management during Ramadan are available, ${ }^{4}$ there are no data in the literature to guide the use of SGLT2 inhibitors during Ramadan. A recent international, retrospective observational study described the characteristics and management of patients with diabetes during Ramadan in 2010 and identified the need for research to explore physicians' beliefs in order to better inform evidence-based management. ${ }^{5} \mathrm{~A}$ systematic review and meta-analysis of other diabetes therapies, including GLP-1 agonists and DPP-4 inhibitors, identified that newer agents cause less hypoglycaemia during Ramadan compared with traditional therapies such as sulphonylureas. ${ }^{6} \mathrm{~A}$ randomised controlled trial, Treat 4 Ramadan, showed that the GLP-1 agonist liraglutide in combination with metformin was safe and effective compared with sulphonylureas during Ramadan. ${ }^{7}$

In advance of Ramadan of 2015, we surveyed the opinions of physicians who manage patients with diabetes on the utility of SGLT2 inhibition at this time; we focused on specialists, but all practising physicians with relevant expertise or knowledge were welcome to participate. The use of SGLT2 inhibitors is expanding rapidly and, in the absence of randomised controlled trials, sharing such information within the medical community may help to guide the use of these agents. Based on our findings and a review of the literature, we present practical guidance on the use of SGLT2 inhibitors in patients with diabetes before, during and after Ramadan. 
Table 1 Summary of survey questions

1. Affirmation of eligibility and consent

2. Specialty

3. Professional appointment (academic/clinical)

4. Professional grade (Consultant, Specialist/Resident, etc.)

5. Identification of given conditions/situations into categories of risk (proposed in the ADA Workshop Report 2010)

6. Identification of given medication-based situations into the categories of risk in patients with type 2 diabetes patients

7. Rate your knowledge/familiarity/experience with the "SGLT2 inhibitors" class of drugs

8. Availability of SGLT2 inhibitors class in ones's practice/country

9. Familiarity with the SGLT2 inhibitors individual drugs

10. Basis of the views on the risks possibly associated with use with the use of "SGLT2 inhibitors" during Ramadan

11. Opinion on the use of the "SGLT2 inhibitors" class of anti-diabetic drugs in patients with type 2 diabetes who choose to fast during Ramadan (general use, no use, selective use)

12. Specific advice to patients

13. Specific advice on the timing of medications (with Iftar vs Sohour)

14. Specific advice on their hydration (intake of extra fluids) in the evening time

15. Specific advice on monitoring their hydration status

16. If, how and when to return to SGLT therapy after Ramadan

The actual questionnaire as used is available on request from the corresponding author.

\section{Methods}

This was a professional survey of the perceptions of doctors regarding the management of patients with type 2 diabetes receiving SGLT2 inhibitor therapy who intend to fast during Ramadan. We used a widely-used web-based commercial survey management service (Survey Monkey, Palo Alto, CA, USA). The questionnaire was formulated de novo based on the objectives of the study, was provided in English only and was mainly in the form of multiple questions with extra options for comments to be added when needed (Table 1). The target population was identified from pooled lists of delegates at recent Continuous Professional Development events, speakers, authors or members of various professional groups or forums. These events took place in various parts of the world but predominantly in the Middle East and North Africa region.

All received an initial email and subsequent reminder emails. A unique email-specific electronic link to the questionnaire was provided. Survey responses were anonymously collected, stored electronically and analysed. Repeat submissions from the same link were automatically blocked by the survey server.

\section{Results}

\section{Characteristics of respondents}

We received 235 responses, mostly from the Middle East and North Africa. Of the 223 eligible respondents, 197 consented to participate.
There were 111 specialists, of whom 62 were endocrinologists. Where grades were reported $(n=162)$, they were mostly consultants (46.3\%) and specialists (31.5\%). Academics represented $13.7 \%$.

\section{Experience, confidence and guidelines}

Respondents reported varying proportions of their patients observing the Ramadan fast: $22.4 \%$ reported $10-25 \%$ of patients, $18.6 \%$ reported $25-50 \%, 18.2 \%$ reported $50-75 \%$ and $23.6 \%$ reported $>75 \%$. Only $12.4 \%$ had more limited experience, with $<10 \%$ of their patients observing the fast. About half $(49.4 \%)$ described themselves as 'fully confident' about the management of diabetes during Ramadan, with lower proportions describing themselves as 'fairly confident' (34.4\%), 'just confident' (11.7\%) or 'unsure' (3.9\%). Common sources of guidance were an ADA workshop report ${ }^{2}$ (used by $54.1 \%$ of respondents) and, to a lesser extent, South Asian Guidelines ${ }^{8}(9.7 \%)$. Many respondents stated that they used common sense and applied principles of good clinical practice $(57.2 \%)$, their own review of the literature (45.9\%) and what they learned from Continuing Medical Education activities (33.3\%).

\section{Recognition of risks during fasting}

The most commonly reported risks of fasting were hypoglycaemia $(98.1 \%)$, dehydration $(83.1 \%)$, hyperglycaemia $(67.5 \%)$, diabetic ketoacidosis (DKA) (63.8\%) and thromboembolic disease (45.2\%). Classifications of various clinical and drug-related scenarios into ADA workshop risk categories are provided in Table 2. There was a poor distinction between the higher risk categories (I and II) and between lower risk categories (III and IV) for several conditions and scenarios (Table 2).

\section{Availability and experience with SGLT2 inhibitors}

Fewer than 50\% of respondents had used SGLT2 inhibitors in their patients with type 2 diabetes. Dapagliflozin was the most widely available SGLT2 inhibitor followed by canagliflozin; very few respondents had access to empagliflozin in their country or practice at the time of the survey, which corresponded with low reports of familiarity of use in the survey.

\section{Physicians' recommendations on the use of SGLT2 inhibitors}

Respondents' views on the use of SGLT2 inhibitors during Ramadan were based on several sources including clinical studies $(22.6 \%)$ or recommendations for use with concomitant agents such as diuretics $(21.1 \%)$, in the presence of renal impairment with and without dialysis (15.8\%) or in the elderly (26.3\%). Some respondents admitted to not being sure of the risks (29.3\%), whereas a substantial proportion of others invoked the 'safety first' rule (35.3\%). A limited number of respondents (6.0\%) indicated some personal experience with their use during fasting. The majority of respondents felt that use of SGLT2 inhibitors was generally appropriate and safe in the management of type 2 diabetes during Ramadan, but that they should be discontinued in selected patients who might be at increased risk of adverse effects (72\%). Endocrinologists appeared to be more cautious, on average, about the use of SGLT2 inhibitors in Ramadan than non-endocrinologists (Table 3). 
Table 2 Physicians' assignments of patients with a given diabetes condition or status (A) or medication-based situations (B) into the ADA categories of risk from fasting during Ramadan

A. Please assign the following condition/situations into the categories of risk in patients with type 1 or type 2 diabetes who fast during Ramadan

\section{Conditions and scenarios}

Severe hypoglycaemia within 3 months prior to Ramadan

All type 1 diabetes patients

A history of recurrent hypoglycaemia

Hypoglycaemia unawareness

Sustained poor glycaemic control $\left(\mathrm{HbA}_{1 \mathrm{c}}>10 \%\right)$

DKA within 3 months prior to Ramadan

Pregnant women

Breastfeeding mothers

$\begin{array}{ll}\text { Very high risk } & \text { High risk } \\ 90(56.9 \%) & 48(31.0) \% \\ 73(47.1 \%) & 66(45.6 \%) \\ 93(60 \%) & 52(33.6 \%) \\ 99(64.7 \%) & 38(24.8 \%) \\ 46(29.7 \%) & 71(45.8 \%) \\ 82(53.3 \%) & 53(34.4 \%) \\ 69(45.1 \%) & 55(35.0 \%) \\ 20(13.5 \%) & 49(33.1 \%)\end{array}$

$\begin{array}{ll}\text { Moderate risk } & \text { Low risk } \\ 13(8.3 \%) & 4(2.6 \%) \\ 16(10.3 \%) & 0(0 \%) \\ 10(6.9 \%) & 0(0 \%) \\ 13(8.5 \%) & 3(2.0 \%) \\ 30(19.4 \%) & 8(5.2 \%) \\ 15(9.7 \%) & 4(2.6 \%) \\ 23(15.0 \%) & 6(3.9 \%) \\ 57(38.5 \%) & 22(15.9 \%)\end{array}$

B. Please assign the following medication-based situations into the categories of risk in patients with type 2 diabetes who fast during Ramadan ${ }^{a}$

\begin{tabular}{|c|c|c|c|c|}
\hline Medication-based situations & Very high risk & High risk & Moderate risk & Low risk \\
\hline Diet only & $4(2.6 \%)$ & $5(3.3 \%)$ & $14(9.1 \%)$ & $131(85.1 \%)$ \\
\hline Metformin only & $6(4.0 \%)$ & $7(4.6 \%)$ & $25(16.5 \%)$ & $114(75 \%)$ \\
\hline Modified-release SUb & $13(8.6 \%)$ & $51(33.8 \%)$ & $71(47.0 \%)$ & $16(10.6 \%)$ \\
\hline Metformin + DDP-4 inhibitor & $3(2.0 \%)$ & $7(4.8 \%)$ & $67(47.0 \%)$ & $70(47.6 \%)$ \\
\hline Metformin and GLP-1 agonist therapy & $4(2.8 \%)$ & $14(9.7 \%)$ & $66(45.5 \%)$ & $61(42.1 \%)$ \\
\hline Metformin and glinides & $8(5.5 \%)$ & $45(31.0 \%)$ & $76(52.4 \%)$ & $16(11 \%)$ \\
\hline$\alpha$-Glucosidase inhibitor only & $3(2.1 \%)$ & $13(9.1 \%)$ & $37(25.9 \%)$ & $90(62.4 \%)$ \\
\hline Metformin and pioglitazone & $2(1.4 \%)$ & $20(13.5 \%)$ & $58(39.2 \%)$ & $68(46.0 \%)$ \\
\hline
\end{tabular}

Table 3 Please indicate your opinion on the use of SGLT2 inhibitors in patients with type 2 diabetes who choose to fast during Ramadan

Possible ways of use/discontinuation of SGLT2
inhibitors in diabetic patients during Ramadan

General use: SGLT2 inhibitors are safe and may be used in all patients with diabetes who intend to observe fasting during Ramadan

No use: SGLT2 inhibitors are not safe and should not be used in any patient with diabetes who intends to observe Ramadan fasting

Selective use ${ }^{\text {b: }}$ SGLT2 inhibitors may generally be safe in patients with diabetes who intend to observe Ramadan fasting, but should be discontinued for selected patients

$$
\text { All }(n=136)
$$

$22 / 136$

$(16.2 \%)$

$18 / 136$

$(13.2 \%)$

$96 / 136$

$(70.6 \%)$
Numbers (\%) of respondents Non-endocrinologists $(n=49)$

$13 / 49$

$(26.5 \%)$

$7 / 49$

$(14.3 \%)$

$29 / 49$

$(59.2 \%)$
Endocrinologists $^{\text {a }}(n=87)$

$22 / 87$

$(10.3 \%)$

$18 / 87$

$(11.0 \%)$

$67 / 87$

(77.1\%)

${ }^{a}$ Response rates were significantly different between endocrinologists and non-endocrinologists (chi-square $=6.55$; Fisher's exact test p<0.04). ${ }^{b}$ Common recurring selection factors were dehydration, hypotension, diuretics, renal impairment, elderly.

Practical advice to patients on SGLT2 inhibitors during Ramadan

Of respondents prepared to prescribe SGLT2 inhibitors in type 2 diabetes patients planning to fast during Ramadan, almost all (93.2\%) expressed the need to give specific advice to patients. Only a minority $(6.8 \%)$ stated that there is no need to provide specific advice and patients may take their SGLT2 inhibitor medication as usual. Almost all (92.2\%) would advise patients to take the SGLT2 inhibitor with the first evening meal (Iftar), with a small minority $(6.1 \%)$ advising taking the medication before the last pre-dawn meal (Suhour).

There was strong support for rehydration with clear fluids 
during the evening after a day's fast, with 9.0\% recommending taking an extra $200-250 \mathrm{~mL}, 32.0 \%$ advising $300-450 \mathrm{~mL}$, $19.7 \%$ advising $500-650 \mathrm{~mL}$ and $22 \%$ advising $700-750 \mathrm{~mL}$; $9.8 \%$ thought that no specific advice was needed. Additionally, $17.7 \%$ of respondents would advise observing colour of the urine, $8.3 \%$ advised checking their blood pressure (at home or at the clinic/surgery), $2.5 \%$ advised observing body weight and $19.8 \%$ advised patients to report if they felt dizzy or lightheaded during the daytime. Varying combinations of 2-4 of these actions were recommended by $42.3 \%$ of respondents, but $11.6 \%$ of respondents felt there was no need for any additional monitoring.

Of those who recommended discontinuation of SGLT2 inhibitors during Ramadan, 34.3\% allowed immediate resumption after the end of Ramadan, 9.5\% after a further week and $4.8 \%$ after a further two weeks. However, $25.7 \%$ would wait until the following clinic visit (with either medical or educator personnel). About one-quarter of respondents (23.8\%) questioned the safety of SGLT2 inhibition during Ramadan and would re-evaluate their place in the patient's management plan on an individual basis on their return to clinic.

\section{Discussion}

We believe that this is the first survey to explore physicians' perspectives of SGLT2 inhibitor use during Ramadan. The results of the survey indicate that some, but not all, of the risks of fasting in Ramadan are recognised by specialists providing diabetes care and few respondents had experience with use of SGLT2 inhibitors during fasting. There were widespread differences in the risk categorisation and medical advice provided to patients on SGLT2 inhibitors regarding adequate rehydration and monitoring for complications. Specialists did not provide consistent advice about ongoing treatment with SGLT2 inhibitors after Ramadan to patients.

Older therapies such as sulphonylureas are known to increase hypoglycaemia in fasting patients during Ramadan, ${ }^{9}$ while SGLT2 inhibitors are associated with less hypoglycaemia. hSGLT2 inhibitors are equally effective with sulphonylureas in terms of

\section{Key messages}

- There are no consensus guidelines on SGLT2 inhibitor use during Ramadan

- This survey indicates that physician knowledge and advice to patients on SGLT2 inhibitors during Ramadan is not consistent or evidence-based

- The results will help to inform the development of guidelines on the use of SGLT2 inhibitors for patients who fast at this time

- Use of SGLT2 inhibitors may generally be safe in most patients with diabetes who intend to observe Ramadan fasting, but should be discontinued for selected patients

glycaemic control when added to metformin in head-to-head studies, and provide reductions in weight and blood pressure. .0,11 $^{1}$ However, SGLT2 inhibitors are also associated with dehydration, postural hypotension, and urinary tract and mycotic fungal genital infections in significant numbers of patients. These sideeffects can be minimised by precautions such as maintaining adequate fluid intake, good hygiene and use in appropriate patients. The recent statement from the FDA on the association of SGLT2 inhibitors with euglycaemic DKA in type 2 diabetes must also be remembered when prescribing these agents. ${ }^{2}$

Fasting during Ramadan is associated with an increased risk of medical complications. It is essential that clinicians are aware of these risks when managing patients with type 2 diabetes in order to reduce morbidity and hospitalisation. Patients observing Ramadan who are on new glucose-lowering therapies such as SGLT2 inhibitors need to be given consistent and evidence-based advice on their known side-effects and how to avoid them. In addition, patients must be supported by trained healthcare professionals with adequate and individualised preparation for

Box 1 Practical tips on the use of SGLT2 inhibitors during Ramadan fasting
Before Ramadan: (assess)
- Ensure absence of contraindications e.g. worsening renal function, use of diuretics
- Avoid in the elderly, those at risk of dehydration, patients on antihypertensive medications
- Advise on diabetes management during Ramadan
- Avoid starting SGLT2 inhibitors immediately before Ramadan
During Ramadan: (monitor)
- Take the medication with the first evening meal (Iftar)
- Encourage intake of adequate fluids at night (approximately extra $500 \mathrm{~mL}$ )
- Avoid excessive intake of salt, coffee or tea
- Observe hydration status (volume and colour of urine)
- Observe blood pressure if home monitoring is readily available
- Report symptoms to clinic/surgery
- If in doubt stop medications and conduct an interim diabetes review
After Ramadan: (review)
- Review the patient in clinic or surgery with 4-6 weeks after Ramadan
- Appraise the experiences and outcomes
- Review the risk/benefit ratio of the class on an individual basis
- Review the need or otherwise for an SGLT2 inhibitor and decide on continuation/resumption or discontinuation 
fasting during Ramadan, using written management plans that include guidance on treatment after fasting.

The results of this survey, along with randomised controlled trials and head-to-head studies with other glucose-lowering therapies, will help to inform the evidence base for preparing appropriate clinical guidelines in patients on SGLT2 inhibitors who choose to fast during Ramadan (Box 1).

Authors contributions $S A B$ and MJD conceived the idea and developed the survey questionnaire, SAB conducted the study logistics. All three authors analyzed the data and produced and revised the manuscript.

Conflict of interest All authors have no conflict of interest in relationship to the current research.

Funding Survey monkey software license is held by SAB by an unrestricted grant from DiaEdu Management Consultants of Dubai, UAE.

\section{References}

1. Vasilakou D, Karagiannis T, Athanasiadou E, et al. Sodium-glucose cotransporter 2 inhibitors for type 2 diabetes: a systematic review and metaanalysis. Ann Intern Med 2013;159:262-74.

http://dx.doi.org/10.7326/0003-4819-159-4-201308200-00007

2. US Food and Dug Administration. FDA warns that SGLT2 inhibitors for diabetes may result in a serious condition of too much acid in the blood. Available at http://www.fda.gov/Drugs/DrugSafety/ucm446845.htm (accessed 7 July 2015)

3 European Medicines Agency. SGLT2 inhibitors. Available at http://www.ema.europa.eu/ema/index.jsp?curl=pages/medicines/human/ referrals/SGLT2_inhibitors/human_referral_prac_000052.jsp\&mid=WCOb 01ac05805c516f (accessed 8 July 2015).
4. Al-Arouj M, Assaad-Khalil S, Buse J, et al. Recommendations for management of diabetes during Ramadan: Update 2010. Diabetes Care 2010;33:1895-902. http://dx.doi.org/10.2337/dc10-0896

5. Babineaux SM, Toaima D, Boye KS, et al. Multi-country retrospective observational study of the management and outcomes of patients with type 2 diabetes during Ramadan in 2010 (CREED). Diabet Med 2015;32:81928. http://dx.doi.org/10.1111/dme.12685

6. Gray LJ, Dales J, Brady EM, et al. Safety and effectiveness of non-insulin glucose-lowering agents in the treatment of people with type 2 diabetes who observe Ramadan: a systematic review and meta-analysis. Diabetes Obes Metab 2015;17:639-48. http://dx.doi.org/10.1111/dom.12462

7. Brady EM, Davies MJ, Gray LJ, et al. A randomized controlled trial comparing the GLP-1 receptor agonist liraglutide to a sulphonylurea as add on to metformin in patients with established type 2 diabetes during Ramadan: the Treat 4 Ramadan trial. Diabetes Obes Metab 2014;16:52736. http://dx.doi.org/10.1111/dom.12249

8. Pathan M, Sahay RK, Zargar AH, et al. South Asian Consensus Guideline: use of insulin in diabetes during Ramadan. Indian J Endocr Metab 2012:16:499-502. http://dx. doi.org/10.4103/2230-8210.97992

9. Aravind SR, Al Tayeb K, Ismail SB, et al. Hypoglycaemia in sulphonylureatreated subjects with type 2 diabetes undergoing Ramadan fasting: a five-country observational study. Curr Med Res Opin 2011;27:1237-42. http://dx.doi.org/10.1185/03007995.2011.578245

10. Goring S, Hawkins N, Wygant G, et al. Dapagliflozin compared with other oral anti-diabetes treatments when added to metformin monotherapy: a systematic review and network meta-analysis. Diabetes Obes Metab 2014;16:433-42. http://dx.doi.org/10.1111/dom.12239

11. Cefalu WT, Leiter LA, Yoon KH, et al. Efficacy and safety of canagliflozin versus glimepiride in patients with type 2 diabetes inadequately controlled with metformin (CANTATA-SU): 52 week results from a randomised, double-blind, phase 3 non-inferiority trial. Lancet 2013;382:941-50. http://dx.doi.org/10.1016/S0140-6736(13)60683-2

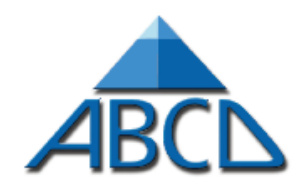

Association of Eritish Clinical Diabetologists

\title{
Canagliflozin (Invokana) Nationwide Audit in progress
}

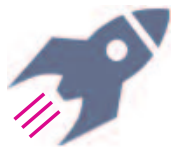

\author{
On January 24th 2016 ABCD launched the Nationwide Audit \\ of canagliflozin in real clinical use in the UK
}

\section{Does your centre use canagliflozin (Invokana)?}

If yes, REGISTER YOUR CENTRE! at http://www.diabetologists-abcd.org.uk/n3/Canagliflozin_Audit.htm

- you are invited to enter your patients' data into the bespoke online tool

- you will be able to analyse your local data easily

- the data will be automatically added to the national data in anonymised form

- we can provide easy-to-complete paper proformas for use in clinic if preferred

Please remember:

- the more data, the more complete our understanding of of canagliflozin in real clinical practice - all contributors will be listed in publications arising from data submission 